\title{
Towards a classification system for errors in trauma resuscitation: is it time?
}

\author{
Kevin High $^{1}$ - Erik P. Hess ${ }^{1}$ (D)
}

Received: 28 May 2021 / Accepted: 5 June 2021

(C) The Author(s), under exclusive licence to Canadian Association of Emergency Physicians (CAEP)/ Association Canadienne de Médecine d'Urgence (ACMU) 2021

In this issue of CJEM, Nikouline and colleagues [1] report the results of a systematic review to identify and codify the types of errors made in adult trauma resuscitation. As reporting of errors in trauma resuscitation often varies between centers with inconsistent use of terminology [2], one potential benefit of the review is to take us closer toward developing a consistent terminology and classification system for errors in trauma resuscitation. This methodologically sound review, which was conducted in accordance with the PRISMA guidelines for reporting items for systematic reviews and meta-analyses [3], identified 19 original research articles meeting inclusion criteria. Detailed review of the included articles identified 39 unique error types, which the authors divided into the following 9 categories: emergency medical services handover, airway, assessment of injuries, patient monitoring and access, transfusion/ blood-related, management of injuries, team communication/dynamics, procedure error, and disposition.

We applaud the authors for a careful review of the published evidence around errors in trauma resuscitation. In our center, a level 1 trauma center in the Southern United States, we have had a system in place for the past 24 years in which all trauma resuscitations, which are collaboratively delivered by emergency physicians and traumatologists, are video recorded. A trauma-trained nurse specialist with quality improvement expertise reviews each case and selects cases to bring to the attention of trauma and emergency medicine leadership. Cases in which errors were made and in which it is thought that there is an opportunity for quality improvement are selected for further review and discussion at our weekly multidisciplinary trauma review conference.

Erik P. Hess

erik.hess@vumc.org

1 Department of Emergency Medicine, Vanderbilt University Medical Center, 1313 21st Avenue South, 703 Oxford House, Nashville, TN 37323-4700, USA
Our video recording system and review process allow for the capture of errors, whether major or minor, early on in the process and provides an avenue for quick mitigation. The system also eliminates any chance of recall bias and provides an accurate record of the resuscitation.

At this hour-long conference, in which residents and attendings in emergency medicine and trauma surgery consistently attend, along with advanced practice providers and nursing staff, the videos are reviewed, errors identified, and both system and individual-level approaches to preventing future errors are discussed. It is with this lens of experience that we read with interest the nine classes of error types proposed by the authors and offer our perspective.

The authors noted significant heterogeneity in both how errors were reported and classified. A portion of this heterogeneity can be attributed to variations in practice, existing systems, and protocols. Further development of this classification system, along with additional descriptors for each category, could help mitigate this phenomenon. We propose two additional categories for consideration and suggest modifications for the transfusion/blood-related and airway management categories (see Table 1).

The trauma resuscitation quality classification system should include an additional category for issues or events around staff safety and safe practice during the resuscitation. This category would include blood and body fluid exposure, sharps injury, and violence towards staff. A sharps exposure during a resuscitative thoracotomy, for example, would fall into this category. Inadequate protective personal equipment compliance by staff, a factor protecting providers from blood and body fluid exposure, should also be incorporated in this category. The classification system should also include an additional category for systems issues that arise from either deviation from existing protocols or system-specific processes. The descriptors for this category should include delays in care along with events falling outside time metrics. For example, a delay in EMS transfer to a level 1 trauma center for a gunshot wound to the torso, or a 
Table 1 Additional categories and suggested modifications to the proposed classification system of trauma errors proposed by Nikouline et al. [1]

\begin{tabular}{ll}
\hline Category & Topics \\
\hline $\begin{array}{l}\text { Staff safety and safe practice } \\
\text { Systems issues due to deviations from system-specific processes or } \\
\text { protocols }^{\mathrm{a}}\end{array}$ & $\begin{array}{l}\text { Blood and body fluid exposure, sharps injury, violence towards staff } \\
\text { Delays in care, other events falling outside time metrics }\end{array}$ \\
$\begin{array}{l}\text { Transfusion/blood-related } \\
\text { b }\end{array}$ & $\begin{array}{c}\text { System specific errors (e.g., adherence to protocol, adherence to } \\
\text { institutional policy) }\end{array}$ \\
Airway $^{\mathrm{b}}$ & Failed airway, surgical airway, hypoxic events
\end{tabular}

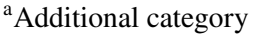

${ }^{\mathrm{b}}$ Suggested modifications to category proposed by Nikouline et al.[1]

delay in establishing intravenous access in a patient leading to delayed CT scanning in a blunt trauma patient could be included in this category.

The transfusion/blood-related category should also include errors specific to the respective system, with specific attention to adherence to protocol and adherence to institutional policy.

The authors noted during their review that errors were more likely to occur in airway management and appropriately designated a category for the same [4, 5]. Given the significance that airway management plays in trauma resuscitation, this category should also include errors due to events such as a failed airway, the need for surgical airway, and hypoxic events.

The authors' efforts towards building an error taxonomy provide an excellent framework on which to build a consistent classification system and terminology for errors in trauma resuscitation. Such a framework would enable comparison between systems and enable progress to be made toward building a safer, higher quality trauma system for patients. We recommend two additional categories along with descriptors for the same as well as developing a tool that is institution and system specific. From our perspective, it is time to have a broader conversation around developing a common classification system for trauma errors to improve quality and safety in trauma resuscitation.

\section{Declaration}

Conflict of Interest None of the authors have any conflicts of interest to declare.

\section{References}

1. Nikouline A, Quirion A, Jung J, Nolan B. Errors in adult trauma resuscitation: a systematic review. CJEM. 2021. https://doi.org/ 10.1007/s43678-021-00118-7.

2. Settervall C, Domingues C, Sousa R, Nogueira L. Preventable trauma deaths. Rev Saude Publica. 2012;46:367-75.

3. Page MJ, McKenzie JE, Bossuyt PM, Boutron I, Hoffmann TC, Mulrow CD, et al. The PRISMA 2020 statement: an updated guideline for reporting systematic reviews. BMJ. 2021;372: n71. https://doi.org/10.1136/bmj.n71.

4. Schoeneberg C, Schilling M, Hussmann B, Schmitz D, Lendemans S, Ruchholtz S. Preventable and potentially preventable deaths in severely injured patients: a retrospective analysis including patterns of errors. Eur J Trauma Emerg Surg. 2017;43(4):481-9.

5. Montmany S, Pallisera A, Rebasa P, Campos A, Colilles C, Luna A, Navarro S. Preventable deaths and potentially preventable deaths. What are our errors? Injury. 2016;47(3):669-73. 